\title{
Numerical and Analytical Calculations for Modeling and Designing Drilling Wicks or Rotary Cutters Based of Functionally Graded Materials
}

\author{
Luc Leroy Mambou Ngueyep $\mathbb{D}^{1,2}$ Joseph Ndop, ${ }^{1,3}$ Elise Rose Atangana Nkene,, \\ and Jean-Marie Bienvenu Ndjaka $(\mathbb{1})^{1}$ \\ ${ }^{1}$ Laboratory of Material Sciences, Department of Physics, Faculty of Sciences, University of Yaoundé 1, P.O. BOX 812, \\ Yaoundé, Cameroon \\ ${ }^{2}$ Department of Mine, Mineral Processing and Environment, School of Geology and Mining Engineering, \\ University of Ngaoundéré, P.O. BOX 115, Meiganga, Cameroon \\ ${ }^{3}$ Douala Institute of Technology, P.O. BOX, 1623 Douala, Cameroon \\ ${ }^{4}$ Department of Physics, Faculty of Sciences, University of Douala, P.O. BOX 24157, Douala, Cameroon
}

Correspondence should be addressed to Luc Leroy Mambou Ngueyep; mamboulucleroy@gmail.com

Received 2 May 2018; Accepted 1 October 2018; Published 1 November 2018

Academic Editor: Parviz Malekzadeh

Copyright (C) 2018 Luc Leroy Mambou Ngueyep et al. This is an open access article distributed under the Creative Commons Attribution License, which permits unrestricted use, distribution, and reproduction in any medium, provided the original work is properly cited.

\begin{abstract}
Drilling tools or drilling pipes, such as drill bit and mill drill, are often subjected to various forces, including essentially tangential forces, centered on their axis of rotation. The main objective of this work is to find analytical and numerical solutions of the distribution of stress field, deformation, and displacement, when such tools are subjected to such forces. It will be assumed that the instrument in the chosen model in this work is a rotating hollow cylinder constructed from a Functional Graded Material (FGM). Because of the graduation of the FGM, the mechanical and elastic properties such as Young's modulus, density, and Poisson's ratio vary in the radial direction according to a power law function. By choosing that the inhomogeneity parameter is between -0.5 and 0.5 , we have established the differential equation which describes the equilibrium of the hollow cylinder in rotation under an axial load. The calculations performed have allowed finding an analytical solution which was compared with numerical solutions obtained by using the shooting method and the fourth-order Runge-Kutta algorithm. These analytical and numerical results have shown that the values of tangential stresses are greater than the radial stresses. The radial stresses and tangential and vertical stresses progressively increase with the axial force $\mathrm{F}_{\mathrm{z}}$. The force $\mathrm{F}_{\mathrm{z}}$ affects more tangential stresses that the radial stresses. The tangential stress, tangential deformations, and displacements are higher on the inner walls of the cylinder than on the exterior surfaces. The results obtained are very important and can be applied in the modeling and designing wicks and drilling strawberries in order to reduce their rapid wear and damage.
\end{abstract}

\section{Introduction}

In exploration and drilling activities, a large majority of mining companies are often confronted with the rapid wear and premature deterioration of drilling wicks, cutter, and strawberries. This problem is mainly due first to the tenacity of media to drill, but then also to the alternation of different backgrounds and the diversity of characteristics they have. This requires for drilling tools, especially strawberries and wicks, performance adapted to the requirements of the media. The design and construction of such tools are therefore an important and constant challenge. It has long been that use is made of composite materials such as tungsten-carbides, tungsten-copper, or titanium, due to their very high resistance, to manufacture most of the cutting and drilling devices [1]. However devices with a variety of properties to meet the above requirements may be possible by using Functionally Graded Materials (FGM) which have 
already helped in solving such problems in many applications [2]. FGM are else purposely graded with respect to their mechanical respective elastic properties or variation of these properties is present since the density or the elasticity varies depending on materials composition [1]. We must remember that many structural components meet very different operating conditions and, therefore, require materials adapted for performances that vary with location within the component. For example, a kitchen knife needs to be hard just to its cutting edge; however, the material from which it is produced must be particularly strong and hard. Similarly the body of a gear has to be solid, so that its surface should be hard and resistant to wear. The body of a turbine blade must be strong, hard, and resistant to creep, while its outer surface must be refractory and resistant to oxidation [3]. It is therefore believed that even the hollow cylinders, built from FGM although appropriate, can provide solutions to applications with different characteristics and requirements which they are subject to. If one thinks of a drilling wick as a rotating hollow cylinder, one can assume that its walls are built of FGM. Optimizing the design of such a cylinder requires an understanding of the elastoplastic behavior of the material [4]. Many numerical and analytical works have been done on this subject, especially on uniform rotating discs, to predict the stress field, deformations, and rupture, to avoid the risk of damage when the device is subjected to different loading conditions [3-22]. In 2005, Ahmet N. Eraslan et al. [4] obtained in the context of the small deformation theory analytical solutions on flat deformations in elastic tubes operably under pressure grades. In 2011, Hasan Callioglu et al. [5] analyzed the state of the stresses on the rotating annular disc made of Functionally Graded Materials (FGM). Similary in 2011, Hassani et al. [6] obtained distributions of stress and strain components of rotating hyperbolic disks with nonuniform material properties subjected to a power form thermoelastic loading under different boundary conditions by semiexact methods of Liao's homotopy analysis method. In 2012, Roberta Sburlati [7] obtained analytical solutions for thick wall cylinders subjected to internal and external pressure, where the entire wall was made of FGM. In 2013 and 2014 Nejad et al. $[8,9]$ presented a closed-form analytical solution in terms of hypergeometric functions to elastic analysis of exponentially functionally graded stationary discs subjected to internal and external pressures. In 2016 Yildirim [10] investigated the exact elastic response of a rotating disk having a continuously varying hyperbolic thickness profile under different boundary conditions. Both convergenthyperbolic and divergent-hyperbolic disk profiles together with uniform profile are all studied. Power-law grading is used for material gradation pattern. In 2017, Wenlong Shen et al. [11] investigated a new simple mathematical method to predict rock stress around a noncircular tunnel and the method is calibrated and validated with a numerical model. It can be found that the tunnel shapes and polar angles affect the applicable zone of the theoretical model signiïñAcantly and the applicable zone of a rectangular tunnel was obtained using this method. Recently in 2018, E. R. Atangana Nkene et al. [12] studied analytically and numerically strain fields, stress fields, and displacements in a rotating hollow cylinder, whose walls were completely made of Functionally Graded Materials (FGM). Their results showed that tangential stress, tangential strain, and displacements are higher at the inner surface and internal radial pressure strongly affects the radial stresses and radial strain.

However, to the best of our knowledge, theoretical and numerical research on deformation states and displacement in a hollow inhomogeneous rotating cylinder subjected to an axial load, as it is the case for strawberries and drill wicks, remain unaddressed. In this work we use numerical and analytical calculations for modeling and designing drilling wicks or rotary cutters based of Functionally Graded Materials.

The paper is organized as follows. Modelisation of a drilling device made of FGM is firstly presented. Analytical equations obtained from modelisation are presented and solved in Section 3. Section 4 presents numerical and analytical results, and then the work is concluded in Section 5.

\section{Method}

2.1. Modelisation of a Drilling Device Made of FGM. Let us consider a drilling device as a hollow FGM circular cylinder with length $\mathrm{L}$ and subjected to the action of a uniform axial loading $F_{z}$. The inner radius and the outer radius of the hollow cylinder are, respectively, $a$ and $b$, as shown in Figure 1.

In this paper we assume that the material is linearly elastic and isotropic and its Young's Modulus depends only on the radial direction. According to Horgan we assume that elastic modulus $E$ and density $\rho$ are given by a power law form function [15]:

$$
\begin{aligned}
& E(r)=E_{0}\left(\frac{r}{b}\right)^{n} ; \\
& \rho(r)=\rho_{0}\left(\frac{r}{b}\right)^{n},
\end{aligned}
$$

with $E_{0}$ and $\rho_{0}$ are reference values of $E$ and, respectively, $\rho, b$ is the outer radius, $r$ is the radial coordinate, and the exponent $n$ can be a positive or a negative real number reflecting the degree of nonuniformity of the material.

Let us consider as in [8-14] that, due to the axisymmetric load condition of the drilling device, the circumferential component of the displacement is zero, and the radial component $u$ depends on the radial distance $r$ only correspondingly; the radial and circumferential components of the stresses $\sigma_{r}$ and $\sigma_{\theta}$ are also $r$-dependent only. We suppose that the length L of the drilling device is very high compared to its radius.

2.2. Stress and Strain Analysis. The second Newton's Law applied to system presented in Figure 1 gives the following equations:

$$
\begin{array}{r}
\frac{\partial \sigma_{r z}}{\partial r}+\frac{1}{r} \frac{\partial \sigma_{\theta z}}{\partial \theta}+\frac{\partial \sigma_{z z}}{\partial z}+\frac{\sigma_{r z}}{r}+\rho F_{z}=0 \\
\frac{\partial \sigma_{r}}{\partial r}+\frac{1}{r} \frac{\partial \sigma_{\theta r}}{\partial \theta}+\frac{\partial \sigma_{z r}}{\partial z}+\frac{\sigma_{r}-\sigma_{\theta}}{r}+\rho F_{r}=0 \\
\frac{\partial \sigma_{r \theta}}{\partial r}+\frac{1}{r} \frac{\partial \sigma_{\theta}}{\partial \theta}+\frac{\partial \sigma_{z \theta}}{\partial z}+\frac{2 \sigma_{r \theta}}{r}+\rho F_{\theta}=0
\end{array}
$$




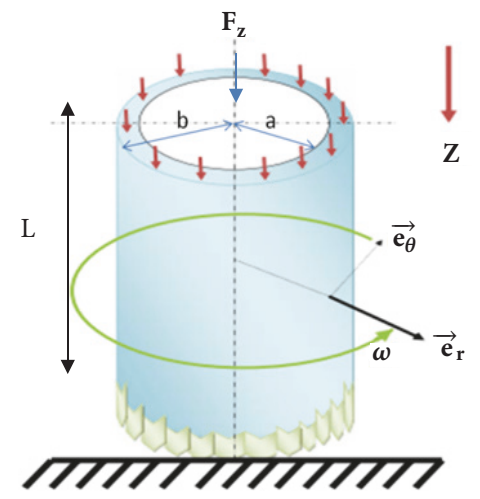

FIGURE 1: A drilling device made of FGM subjected to the action of a uniform axial load $F_{z}$.

By considering a drilling device an axisymmetric problem, (2) becomes

$$
\begin{aligned}
\frac{d}{d r}\left(r \sigma_{r}\right)-\sigma_{\theta}+\rho(r) \omega^{2} r^{2} & =0 \\
\frac{d \sigma_{z}}{d z}+\rho g+\frac{F_{z}}{L} & =0
\end{aligned}
$$

$F_{z}$

where $\omega$ is the angular velocity of cylinder, $\rho g$ is the body force of the material per unit volume, and $F_{z}$ is external mechanical load. By taking account of the small deformations and due to the rotational symmetry, the strain-displacement relations become

$$
\begin{aligned}
\varepsilon_{r} & =\frac{d u_{r}}{d r} ; \\
\varepsilon_{\theta} & =\frac{u_{r}}{r} ; \\
\gamma_{r \theta} & =0
\end{aligned}
$$

By applying Hookes' Law to system (see Figure 1), relations of stress-strain are given by [12]

$$
\begin{aligned}
& \sigma_{r}=\frac{E_{0}}{(1+\nu)(1-2 v)}\left(\frac{r}{b}\right)^{n}\left[\nu \varepsilon_{z}+\frac{v u_{r}}{r}+(1-v) \frac{d u_{r}}{d r}\right] \\
& \sigma_{\theta}=\frac{E_{0}}{(1+\nu)(1-2 v)}\left(\frac{r}{b}\right)^{n}\left[\nu \varepsilon_{z}+\frac{(1-\nu) u_{r}}{r}+\nu \frac{d u_{r}}{d r}\right] \\
& \sigma_{z}=E_{0}\left(\frac{r}{b}\right)^{n} \varepsilon_{z}+\nu\left(\sigma_{r}+\sigma_{\theta}\right)
\end{aligned}
$$

where $v$ is Poisson's Coefficient

Substituting (5) into (3), the equation of displacement is given by [12]

$$
\begin{gathered}
r \frac{d^{2} u_{r}}{d r^{2}}+(n+1) \frac{d u_{r}}{d r}+\frac{[\nu(n+1)-1]}{r(1-v)} u_{r}+\frac{n v \varepsilon_{z}}{1-\nu} \\
+\frac{(1+v)(1-2 v) \rho_{0} \omega^{2}}{E_{0}(1-v)} r^{2}=0
\end{gathered}
$$

This equation can be written as

$$
\begin{gathered}
r^{2} \frac{d^{2} u_{r}}{d r^{2}}+(n+1) r \frac{d u_{r}}{d r}-\frac{[1-v(n+1)]}{(1-v)} u_{r} \\
=-\frac{n r v \varepsilon_{z}}{1-v}-\frac{(1+v)(1-2 v) \rho_{0} \omega^{2}}{E_{0}(1-v)} r^{3}
\end{gathered}
$$

The next section is devoted to solving analytically and numerically (7).

\section{Analytical and Numerical Solution}

3.1. Analytical Solution. Analytical solution of (7) is given as in $[12]$ by

$$
u(r)=K_{1} r^{-(n+K) / 2}+K_{2} r^{-(n-K) / 2}+A r+D r^{3}
$$

where $K=\sqrt{n^{2}+4-4 n v /(1-\nu)} ; A=-\nu \varepsilon_{z} ; D=-(1+\nu)(1-$ 2v) $\rho_{0} \omega^{2} /[8+3 n-2(4+n) \nu] E_{0}$; and $K_{1}$ and $K_{2}$ are the constants of integration.

In the case of homogenous material, (8) becomes

$$
\begin{aligned}
& u_{r}=K_{1} r^{-1}+K_{2} r-\nu \varepsilon_{z} r ; \quad n=0 ; \omega=0 \\
& \begin{array}{r}
u_{r}=K_{1} r^{-1}+K_{2} r-\frac{(1+\nu)(1-2 \nu) \rho_{0} \omega^{2}}{8(1-\nu) E_{0}} r^{3}-\nu \varepsilon_{z} r ; \\
n=0 ; \omega \neq 0
\end{array}
\end{aligned}
$$

By combining (8) and (4), we obtain the components of strain tensor as in [12]:

$$
\begin{aligned}
\varepsilon_{r}= & -K_{1} \frac{(n+K)}{2} r^{-(n+K+2) / 2}-K_{2} \frac{(n-K)}{2} r^{-(n-K+2) / 2} \\
& -\frac{3(1+\nu)(1-2 \nu) \rho_{0} \omega^{2}}{[8+3 n-2(4+n) \nu] E_{0}} r^{2}-\nu \varepsilon_{z} \\
\varepsilon_{\theta}= & K_{1} r^{-(n+K+2) / 2}+K_{2} r^{-(n-K+2) / 2} \\
& -\frac{(1+\nu)(1-2 \nu) \rho_{0} \omega^{2}}{[8+3 n-2(4+n) \nu] E_{0}} r^{2}-\nu \varepsilon_{z}
\end{aligned}
$$


By combining (10) and (5), we obtain the components of stress tensor as in [12]:

$$
\begin{aligned}
& \sigma_{r}=-\frac{E_{0}}{2(1+\nu)(1-2 \nu) b^{n}} \\
& \cdot r^{(n-K-2) / 2}\left\{K_{1}[(K+n)(1-\nu)-2 \nu]\right. \\
& \left.+K_{2} r^{K}[(n-K)(1-v)-2 \nu]\right\} \\
& -\frac{(3-2 v) \rho_{0} \omega^{2}}{8+3 n-2 v(4+n) b^{n}} r^{n+2} \\
& \sigma_{\theta}=-\frac{E_{0}}{2(1+\nu)(1-2 \nu) b^{n}} \\
& \cdot r^{(n-K-2) / 2}\left\{K_{1}[-2+(K+n+2) \nu]\right. \\
& \left.+K_{2} r^{K}[-2+(n-K+2) \nu]\right\} \\
& -\frac{[1+2 \nu] \rho_{0} \omega^{2}}{8+3 n-2 \nu(4+n) b^{n}} r^{n+2} \\
& \sigma_{z}=-\frac{v E_{0}}{2(1+\nu)(1-2 \nu) b^{n}} r^{(n-K-2) / 2}\left\{K_{1}(K+n-2)\right. \\
& \left.+K_{2}(K-n-2) r^{K}\right\}-\frac{4 \nu \rho_{0} \omega^{2}}{8+3 n-2 \nu(4+n) b^{n}} r^{n+2} \\
& +\frac{r^{n} \varepsilon_{z} E_{0}}{b^{n}}
\end{aligned}
$$

The Boundary Conditions. In this part we use the mechanical boundary conditions to determine the constants $K_{1}$ and $K_{2}$. It can be expressed as

$$
\begin{aligned}
& \sigma_{r}(b)=0 ; \\
& \sigma_{r}(a)=0
\end{aligned}
$$

By substituting (12) into (11), the constants are given by

$$
\begin{aligned}
& K_{1} \\
& =-\frac{2 K_{11}(1+v)(1-2 v)}{[8+3 n-2 v(4+n)][(n+K)(1-v)-2 v] E_{0}\left(a^{K}-b^{K}\right)} \\
& K_{2} \\
& =-\frac{2 K_{22}(1+v)(1-2 v)}{[8+3 n-2 v(4+n)][(n-K)(1-v)-2 v] E_{0}\left(a^{K}-b^{K}\right)}
\end{aligned}
$$

where

$$
\begin{aligned}
& K_{11} \\
& \quad=\left\{(3-2 \nu)\left(b^{(n+K)} a^{(n+K+6) / 2}-a^{K} b^{(3 n+K+6) / 2}\right)\right\} \rho_{0} \omega^{2} \\
& K_{22}=\left\{(3-2 \nu)\left(a^{(n+K+6) / 2} b^{n}-b^{(3 n+K+6) / 2}\right)\right\} \rho_{0} \omega^{2}
\end{aligned}
$$

In the case of homogenous drilling device, (13) becomes

$$
\begin{aligned}
& K_{1}=\frac{\left[\left(b^{2}-a^{2}\right)(3-2 \nu) \rho \omega^{2}\right](1+v) a^{2} b^{2}}{8\left(a^{2}-b^{2}\right)(v-1) E_{0}} \\
& K_{2}=-\frac{\left[\left(b^{2}-a^{2}\right)(3-2 \nu) \rho \omega^{2}\right](1+v)(2 v-1)}{8\left(a^{2}-b^{2}\right)(\nu-1) E_{0}} ; \\
& n=0, \omega \neq 0
\end{aligned}
$$

In order to determine the constant $\varepsilon_{z}$, we use the expression of the $Z$ component the force $F_{z}$ given by

$$
\begin{aligned}
F_{z}^{(\alpha, \beta)}= & \int_{\alpha}^{\beta} r \sigma_{z} d r \\
= & B_{1}\left[\alpha^{(n-K+2) / 2}-\beta^{(n-K+2) / 2}\right] \\
& +B_{2}\left[\alpha^{(n+K+2)}-\beta^{(n+K+2)}\right] \\
& +B_{3}\left[\alpha^{(n+4)}-\beta^{(n+4)}\right] \\
& +B_{4}\left[\alpha^{(n+2)}-\beta^{(n+2)}\right] \varepsilon_{z}
\end{aligned}
$$

where

$$
\begin{aligned}
& B_{1}=-\frac{v E_{0}(K+n-2) C_{1}}{2(1+\nu)(1-2 \nu) b^{n}} ; \\
& B_{2}=-\frac{v E_{0}(K-n-2) C_{2}}{2(1+\nu)(1-2 \nu) b^{n}} ; \\
& B_{3}=-\frac{4 \nu \rho_{0} \omega^{2}}{8+3 n-2 \nu(4+n) b^{n}} ; \\
& B_{4}=\frac{E_{o}}{b^{n}}
\end{aligned}
$$

As it is the case in drilling devices or drilling systems, we suppose that the rotating hollow cylinder is subjected to a hydraulic force $F_{z}$. Let assume that this force is constant. By solving (16) we obtain

$$
\begin{aligned}
\varepsilon_{z}= & \frac{(n+2) F_{z}}{B_{4}}\left[\alpha^{-(n+2)}-\beta^{-(n+2)}\right] \\
& -\frac{2(n+2) B_{1}}{(n-K+2) B_{4}}\left[\alpha^{-(n+K+2) / 2}-\beta^{-(n+K+2) / 2}\right] \\
& -\frac{2(n+2) B_{2}}{(n+K+2) B_{4}}\left[\alpha^{-(n-K+2) / 2}-\beta^{-(n-K+2) / 2}\right] \\
& -\frac{(n+2) B_{3}}{(n+4) B_{4}}\left[\alpha^{2}-\beta^{2}\right]
\end{aligned}
$$

where $\alpha=\mathrm{a}$ (inner radius) and $\beta=\mathrm{b}$ (outer radius).

3.2. Numerical Solution. In the next part, we now study the influence of the force $F_{z}$ and an inhomogeneous parameter $n$ 
on the stress, strain, and displacement in the hollow cylinder. We use therefore the constants given by [15]

$$
\begin{aligned}
a & =0.08 \mathrm{~m} ; \\
b & =0.1 \mathrm{~m} ; \\
\nu & =0.3 ; \\
E_{0} & =420.0 * 10^{9} \mathrm{~Pa} ; \\
\rho_{0} & =15800 \mathrm{Kg} \cdot \mathrm{m}^{-3} ; \\
\omega & =250 \mathrm{rad} . \mathrm{s}^{-1} ; \\
a & \leq r \leq b ; \\
L & =10 \mathrm{~m}
\end{aligned}
$$

Numerical Schemes. Let consider (7):

$$
\begin{gathered}
r^{2} \frac{d^{2} u_{r}}{d r^{2}}+(n+1) r \frac{d u_{r}}{d r}-\frac{[1-\nu(n+1)]}{(1-\nu)} u_{r} \\
=-\frac{n r \nu \varepsilon_{z}}{1-\nu}-\frac{(1+\nu)(1-2 v) \rho_{0} \omega^{2}}{E_{0}(1-v)} r^{3}
\end{gathered}
$$

By applying the boundary conditions

$$
\begin{aligned}
& u(a)=0 ; \\
& u(b)=0
\end{aligned}
$$

(7) can be transformed as

$$
\begin{aligned}
Y_{2}^{\prime}= & -\frac{1+n}{r} Y_{2}+\frac{1-\nu(n+1)}{(1-\nu) r^{2}} Y_{1} \\
& -\frac{\rho_{0} \omega^{2} r(1+\nu)(1-2 \nu)}{b^{n} E_{0}(1-\nu)}-\frac{n r \nu \varepsilon_{z}}{r(1-\nu)}
\end{aligned}
$$

where $Y_{1}=u$ and $Y_{2}=d u / d r=u^{\prime}=f\left(r, Y_{1}, Y_{2}\right)$.

Equation (22) is transformed into a set of differential equations that are solved using the shooting method and the fourth-order Runge-Kutta algorithm. The analytical and numerical solutions obtained are thus presented.

\section{Numerical and Analytical Results}

In Figures 2, 3, 4, 5, 6, and 7, the numerical results are plotted in green triangle and analytical results are plotted in red, blue, and black colors, respectively, for $\mathrm{Fz}=10 \mathrm{kN}$, $50 \mathrm{kN}$, and $100 \mathrm{kN}$. In these figures we observe the perfect correlation between analytical results and numerical results. There is no gap between these curves. Then, in this section, comments and analysis made for analytical results are valid for numerical results.

Figure 2 presents the variation of the displacement along the radial direction of the inhomogeneous hollow cylinder submitted to an axial loading.

In Figure 2(a) we see that, for $n=0.5$, the displacement increases gradually with the force $F_{z}$. Figure 2(b) shows that, for $n=-0.5$, the displacement decreases gradually with increasing the force $F_{z}$ and has its lowest value when $F_{z}$ reaches its highest value. For $n=0.5$ (greater than zero), the highest value of $F_{z}$ gives the highest value of the displacement. In addition, according to the boundary conditions, the radial displacements are zero at the inner and outer surfaces. From $r=0.08 \mathrm{~m}$ to $r=0.1 \mathrm{~m}$, the displacement increases gradually and reaches the maximum values $10^{-9} \mathrm{~m}$ and $7.5 \times 10^{-9}$ $m$ at $r=0.9$, respectively, for $n=0.5$ and $n=-0.5$ and then decreases to zero. Moreover the displacements increase when the inhomogeneous parameter $n$ increases. They have lower value for $n=-0.5$ when compared with the results for $n=0,5$.

Figure 3 presents the variation of radial strains along the radial direction of an inhomogeneous hollow cylinder submitted to an axial loading. We see that the radial strain decreases linearly along the radial direction. All radial strains are higher at the inner surface.

We observe that the force $F_{z}$ does not much affect the radial strain, in particular when $r \approx(a+b) / 2$. In Figure 3(a), for $r=[0.8-0.85]$ radial strains increase when $F_{z}$ increases and for $r=[0.95-1]$ radial strains decrease when $F_{z}$ increases. In Figure 3(b) for $r=[0.8-0.85]$ radial strains decrease when $F_{z}$ increases and for $r=[0.95-1]$ radial strains increase when $F_{z}$ increases. Moreover radial strains increase when the inhomogeneous parameter $n$ increases. They have lower value for $n=-0.5$ when compared with the results for $n=0,5$.

Figure 4 illustrates the variation of the tangential strain along the radial direction of the inhomogeneous hollow cylinder. We can observe that tangential strains $\varepsilon_{\theta}$ are zero at the inner and outer surfaces. From $r=0.08 m$ to $r=0.1 m$, tangential strains gradually increase and reach the maximum values $11.5 \times 10^{-8}$ and $8.5 \times 10^{-8}$ at $r=0.9$, respectively, for $n=$ 0.5 and $n=-0.5$ and then decrease to zero. Moreover they increase when the inhomogeneous parameter $n$ increases, and have lower value for $n=-0.5$ when compared with the results for $n=0.5$. We can see that, in Figure 4(a), for $n=0.5$, tangential strains $\varepsilon_{\theta}$ increase gradually with the force $F_{z}$ and in Figure $4(\mathrm{~b})$, for $n=-0.5$, they decrease gradually with $F_{z}$. For $n=-0.5$ (less than zero), the highest value of $F_{z}$ gives the lowest value of strain, and for $n=0.5$ (greater than zero) the highest value of $F_{z}$ gives the highest value of tangential strain.

We note in Figure 5 that the vertical stresses vary linearly along the radial direction of the cylinder. For $n=-0.5$, the vertical stresses decrease slowly with radius ( $r$ ) and for $n=0.5$, vertical stresses increase slowly in radial direction. For $n=-$ 0.5 , the maximum values of vertical stresses are $8.6 \mathrm{MPa}$, 4.2 $\mathrm{MPa}$, and $1 \mathrm{MPa}$, respectively, for $F_{z}=100 \mathrm{kN}, 50 \mathrm{kN}$, and $10 \mathrm{kN}$. For $\mathrm{n}=0.5$, the maximum values of vertical stresses are $10 \mathrm{MPa}, 5 \mathrm{MPa}$, and $1 \mathrm{MPa}$, respectively, for $F_{z}=100 \mathrm{kN}$, $50 \mathrm{kN}$, and $10 \mathrm{kN}$. For $\mathrm{n}=-0.5$ and $\mathrm{n}=0.5$, the vertical stresses increase with the force $F_{z}$. In addition the inhomogeneous parameter $n$ does not much affect the vertical stresses.

As can be seen in Figures 6 and 7, radial and tangential stresses decrease linearly along the radial direction. They are higher at the inner surface and vary with parallel curves in the radial direction of the cylinder. In addition the radial and tangential stresses decrease when the inhomogeneous parameter $n$ increases and gradually increase when the force 


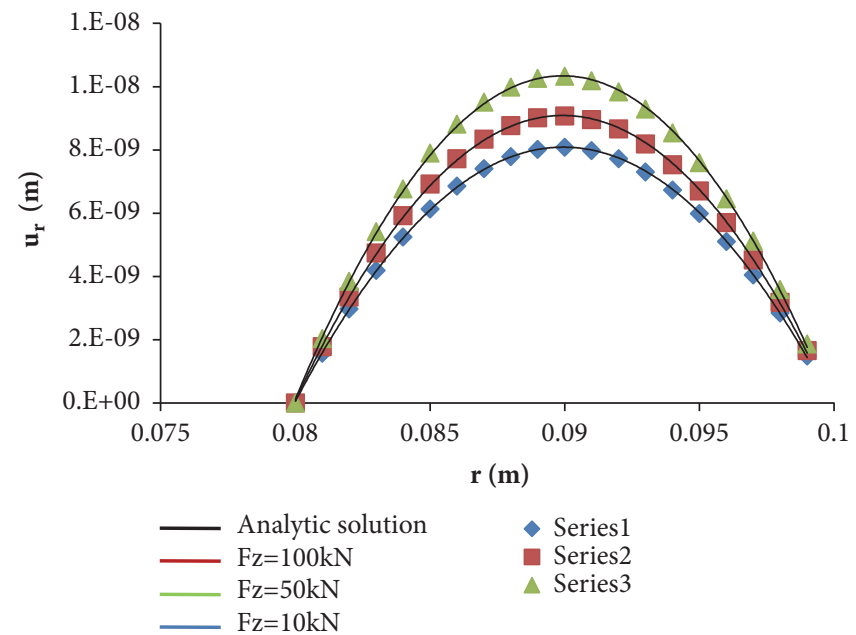

(a) $\mathrm{n}=0.5$
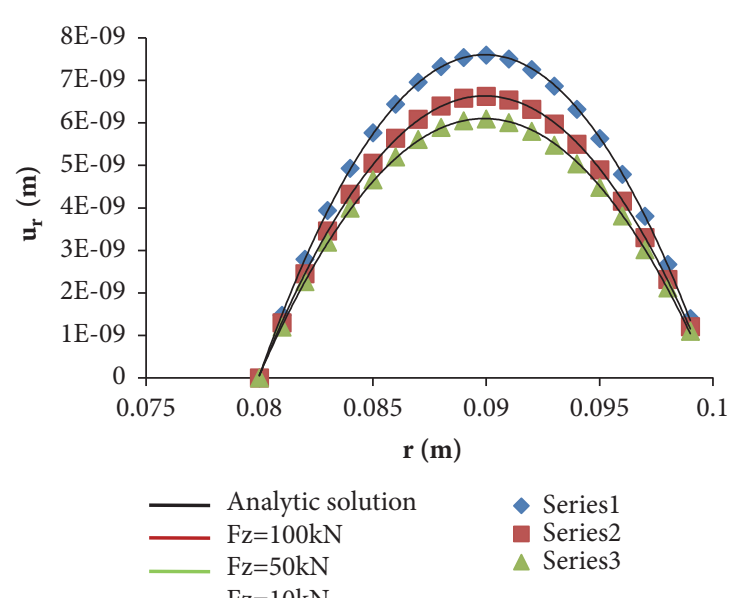

(b) $\mathrm{n}=-0.5$

FIGURE 2: Displacement $u_{r}$ as a function of the radial coordinate $r$ : (a) for $n=0.5$, (b) $n=-0.5$.

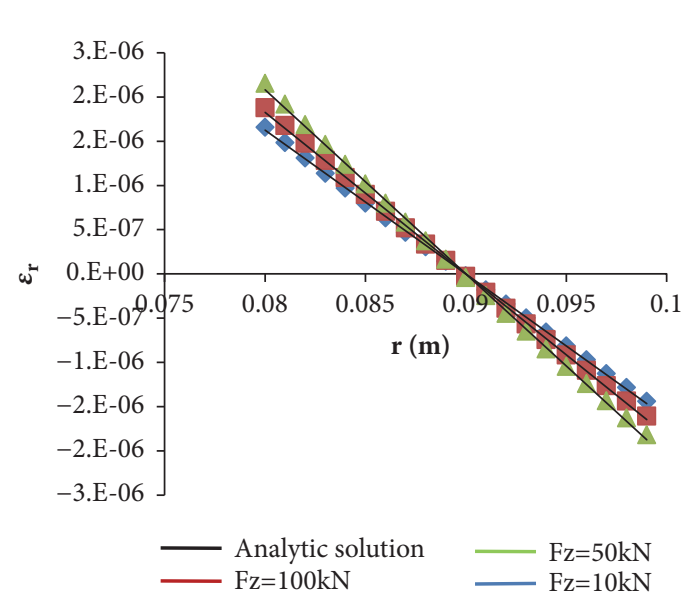

(a) $\mathrm{n}=0.5$
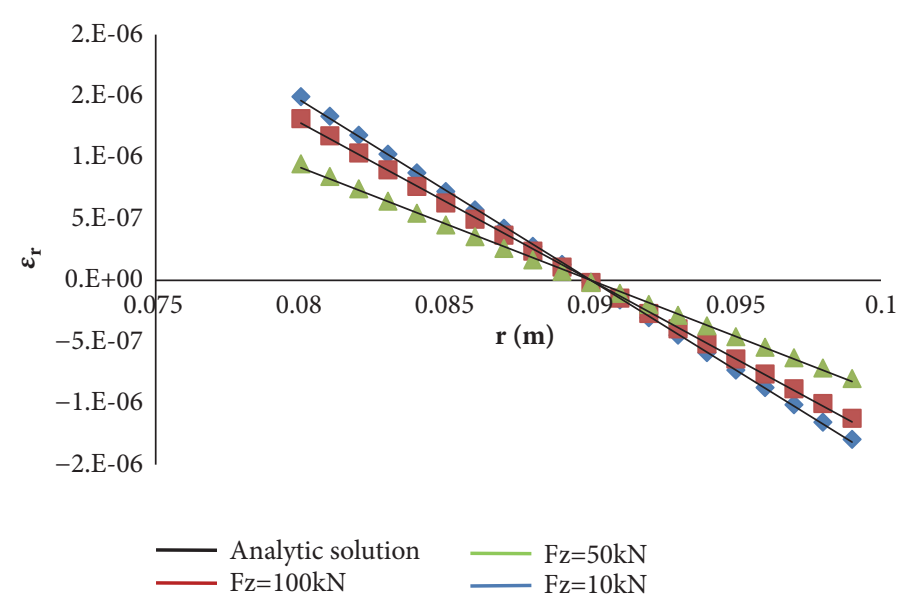

(b) $\mathrm{n}=-0.5$

FIGURE 3: Radial strain $\varepsilon_{r}$ as a function of the radial coordinate $\mathrm{r}$ : (a) for $n=0.5$, (b): $n=-0.5$.

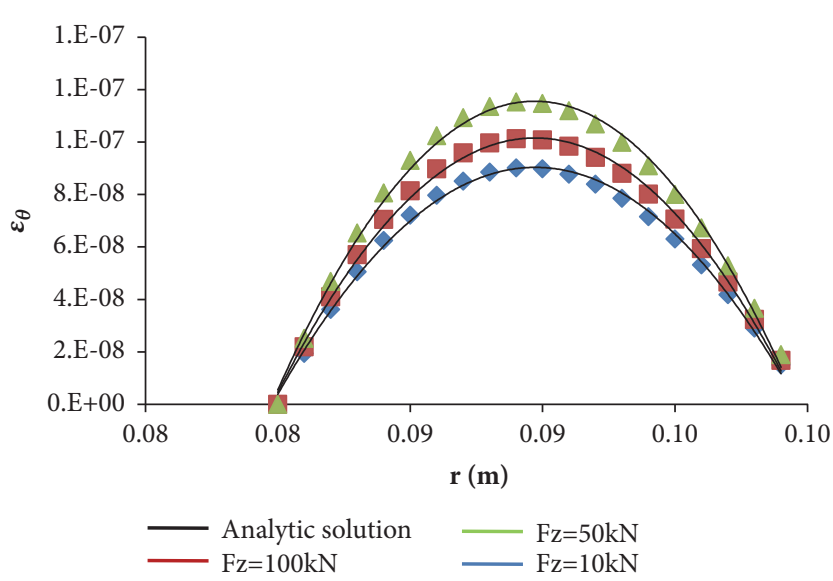

(a) $\mathrm{n}=0.5$

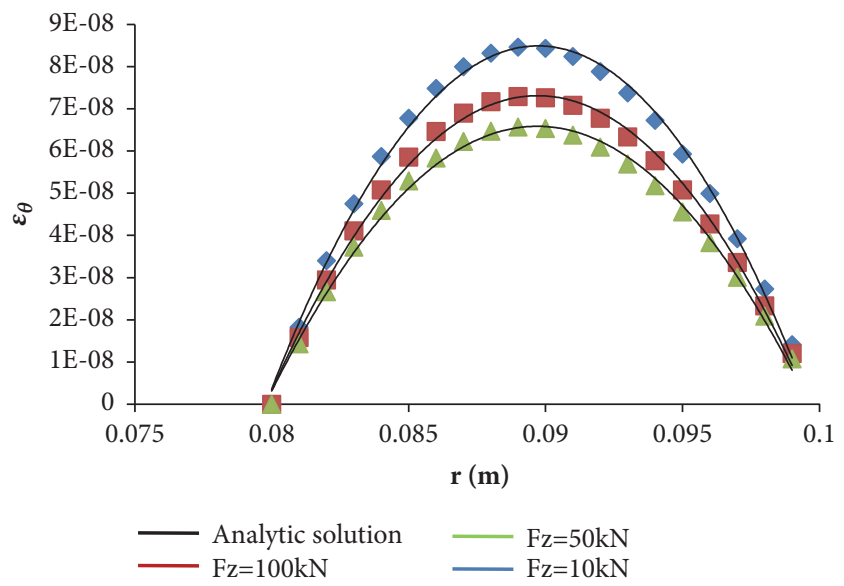

(b) $\mathrm{n}=-0.5$

FIGURE 4: Tangential strain $\varepsilon_{\theta}$ as a function of radial coordinate: (a) for $n=0.5$, (b): $n=-0.5$. 


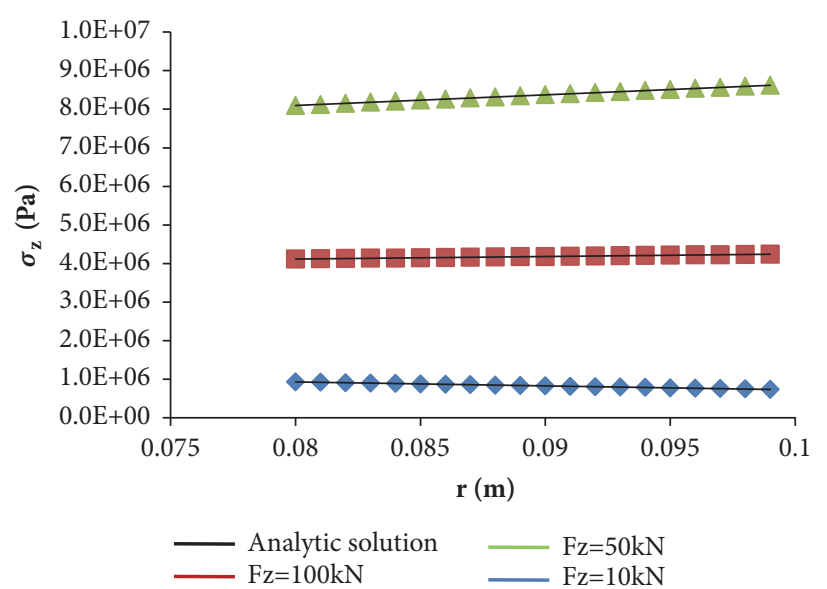

(a) $\mathrm{n}=0.5$

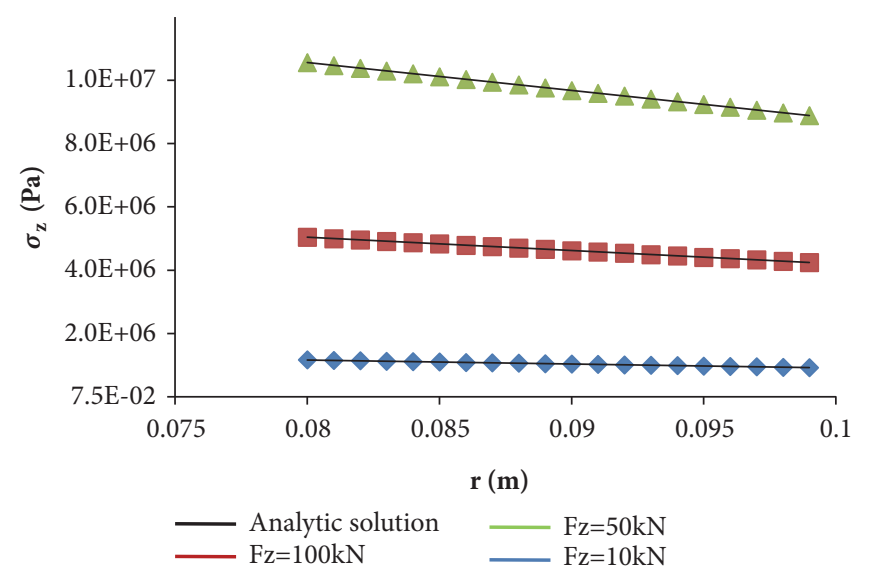

(b) $\mathrm{n}=-0.5$

FiguRE 5: Vertical stress $\varepsilon_{\theta}$ as a function of the radial coordinate $r$ : (a) for $n=0.5$, (b): $n=-0.5$.

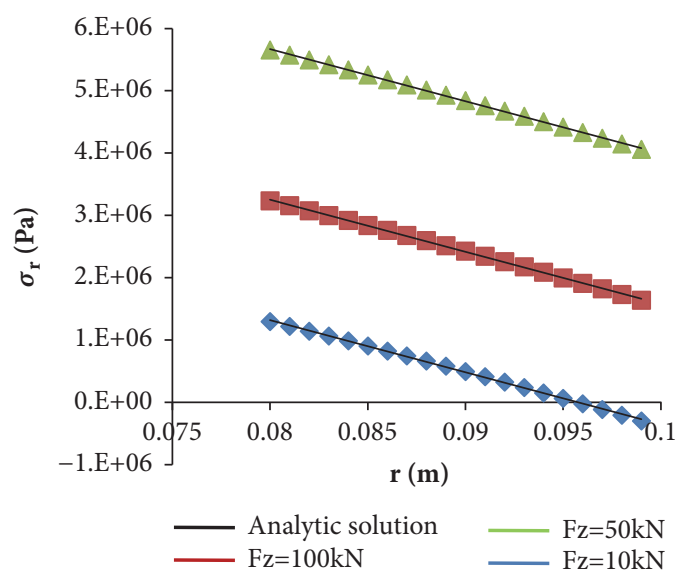

(a) $\mathrm{n}=0.5$

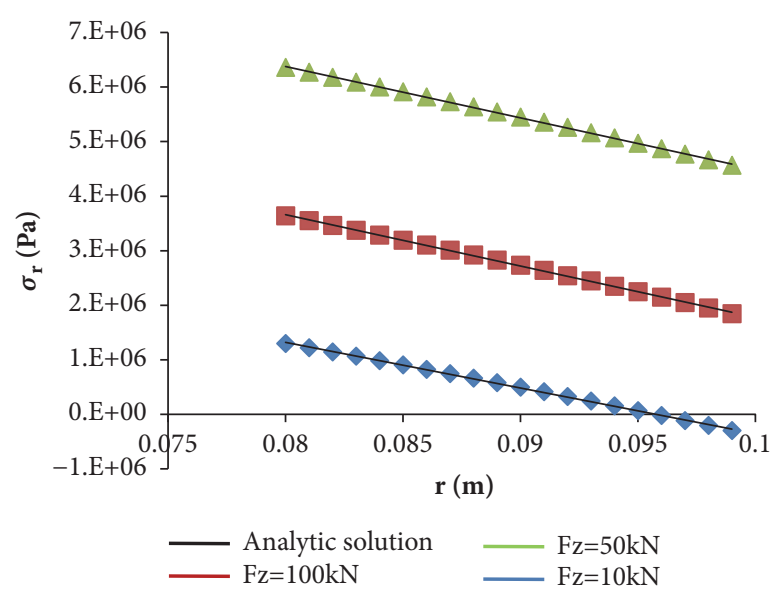

(b) $\mathrm{n}=-0.5$

FIGURE 6: Radial stress $\sigma_{r}$ as a function of the radial coordinate $r$ : (a) for $n=0.5$, (b): $n=-0.5$.

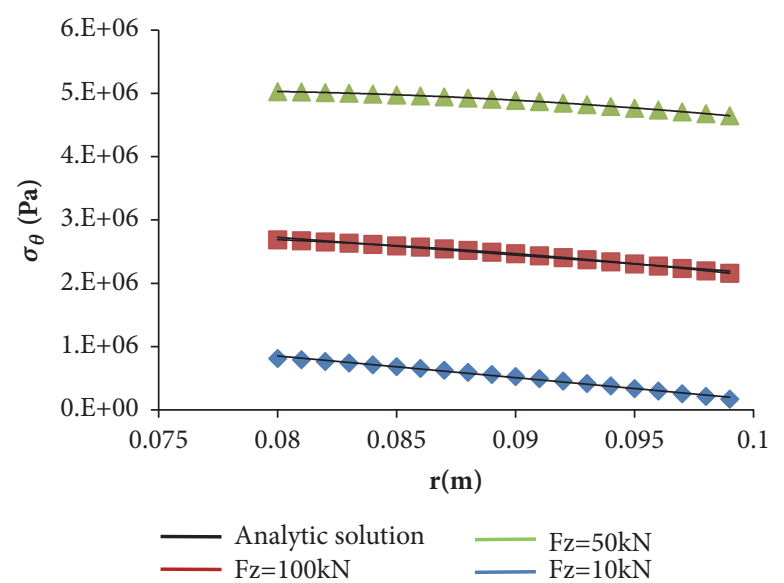

(a) $\mathrm{n}=0.5$

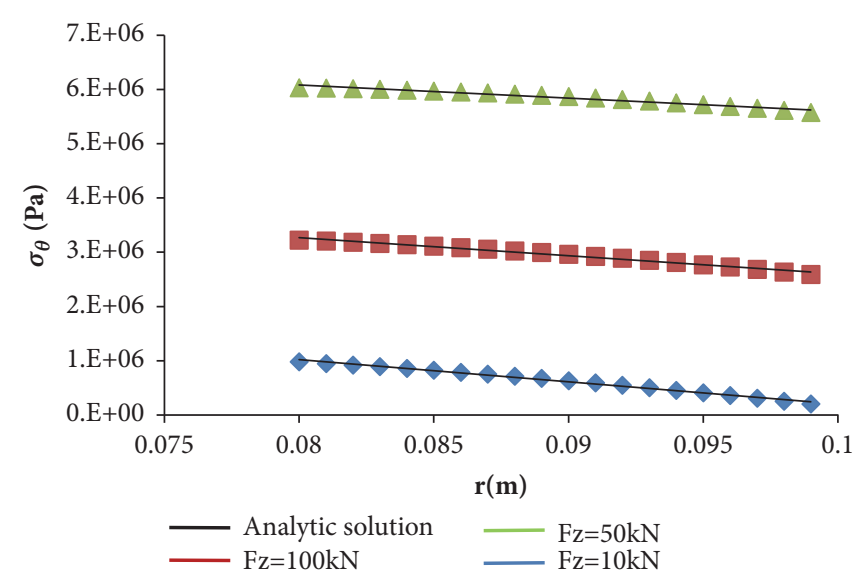

(b) $\mathrm{n}=-0.5$

Figure 7: Tangential stress $\sigma_{\theta}$ as a function of radial coordinate $r$ : (a) for $n=0.5$, (b): $n=-0.5$. 
$F_{z}$ increases. Moreover the radial stresses curves save the parallelism when $F_{z}$ and the inhomogeneous parameter $n$ change.

\section{Conclusion}

In this paper, plane stresses, strains, and displacements along the radial direction of an inhomogeneous hollow rotating cylinder under axial loading have been analyzed. Analytical and numerical results obtained lead us to conclude the following:

(i) The stresses, strains, and displacement obtained from analytical and numerical solutions are in good correlation. Analytical curves and numerical curves are merged. There is no gap between these curves. This confirms the veracity of numerical and analytical results.

(ii) The force $F_{z}$ strongly affects the radial and tangential stresses.

(iii) Vertical stresses do not much change in the radial direction.

(iv) The magnitudes of radial stresses are higher than those of tangential stresses.

(v) Tangential stresses, radial stresses, tangential strain, and displacements are higher at the inner surface.

(vi) The variation of the mechanical and elastic properties, due to the graduation of the Functional Graded Material (FGM), strongly affects the distribution of tangential and radial stresses.

The results obtained in this work are certainly of great importance, as they can contribute to the modeling and designing of drilling strawberry and wicks or rotary cutters.

\section{Data Availability}

The data used to support the findings of this study are available from the corresponding author upon request.

\section{Conflicts of Interest}

The authors declare that they have no conflicts of interest regarding the publication of this paper.

\section{References}

[1] J. Ndop, T. J. Kim, and W. Grill, "Mechanical characterization of graded materials by ultrasonic microscopy with Phase contrast," Materials sciences Forum, vol. 308-311, pp. 873-878, 1999.

[2] J. Ndop, Mechanische Charakterisierung gradierter Materialien mit der Raster-Ultraschallmikroskopie mit Vektorkontrast, Dissertation, Fakultaet fuer Physik und Geowissenschaften, Universitaet Leipzig, Germany, 2000.

[3] S. Suresh and A. Mortensen, Fundamentals of functionally graded materials, Book 698, IOM Communications Ltd.

[4] A. N. Eraslan and T. Akis, "Elastoplastic response of a long functionally graded tube subjected to internal pressure," Turkish
Journal of Engineering and Environmental Sciences, vol. 29, no. 6, pp. 361-368, 2005.

[5] H. Çallioğlu, N. B. Bektaş, and M. Sayer, "Stress analysis of functionally graded rotating discs: Analytical and numerical solutions," Acta Mechanica Sinica, vol. 27, no. 6, pp. 950-955, 2011.

[6] A. Hassani, M. H. Hojjati, G. Farrahi, and R. A. Alashti, "Semi-exact elastic solutions for thermo-mechanical analysis of functionally graded rotating disks," Composite Structures, vol. 93, no. 12, pp. 3239-3251, 2011.

[7] R. Sburlati, "Analytical elastic solutions for pressurized hollow cylinders with internal functionally graded coatings," Composite Structures, vol. 94, no. 12, pp. 3592-3600, 2012.

[8] M. Z. Nejad, M. Abedi, M. H. Lotfian, and M. Ghannad, "Elastic analysis of exponential FGM disks subjected to internal and external pressure," Central European Journal of Engineering, vol. 3, no. 3, pp. 459-465, 2013.

[9] M. Z. Nejad, A. Rastgoo, and A. Hadi, "Exact elasto-plastic analysis of rotating disks made of functionally graded materials," International Journal of Engineering Science, vol. 85, pp. 47-57, 2014.

[10] V. Yildirim, "Analytic solutions to power-law graded hyperbolic rotating discs subjected to different boundary conditions," International Journal of Engineering and Applied Sciences, vol. 8, no. 1, pp. 38-52, 2016.

[11] W. Shen, X. Wang, J. Bai, W. Li, and Y. Yu, "Rock stress around noncircular tunnel: a new simple mathematical method," Advances in Applied Mathematics and Mechanics, vol. 9, no. 6, pp. 1330-1346, 2017.

[12] E. R. Atangana Nkene, L. L. Mambou Ngueyep, J. Ndop, E. S. Djiokeng, and J. B. Ndjaka, "Displacements, strains, and stresses investigations in an inhomogeneous rotating hollow cylinder made of functionally graded materials under an axisymmetric radial loading," World Journal of Mechanics, vol. 08, no. 03, pp. 59-72, 2018.

[13] A. Kursun, E. Kara, E. Çetin, S. Aksoy, and A. Kesimli, "Mechanical and thermal stresses in functionally graded cylinders," International Journal of Mechanical, Aerospace, Industrial, Mechatronic and Manufacturing Engineering, vol. 8, no. 2, 2014.

[14] A. N. Eraslan, "Elastic-plastic deformations of rotating variable thickness annular disks with free, pressurized and radially constrained boundary conditions," International Journal of Mechanical Sciences, vol. 45, pp. 643-667, 2007.

[15] C. O. Horgan and A. M. Chan, "Pressurized hollow cylinder or disk problem for functionally graded isotropic linearly elastic materials," Journal of Elasticity, vol. 55, no. 1, pp. 43-59, 1999.

[16] A. N. Eraslan, "Inelastic deformations of rotating variable thickness solid disks by tresca and von mises criteria," International Journal of Computational Engineering Science, vol. 3, pp. 89-101, 2002.

[17] N. Tutuncu, "Stresses in thick-walled FGM cylinders with exponentially-varying properties," Engineering Structures, vol. 29, no. 9, pp. 2032-2035, 2007.

[18] X.-L. Peng and X.-F. Li, "Thermal stress in rotating functionally graded hollow circular disks," Composite Structures, vol. 92, no. 8, pp. 1896-1904, 2010.

[19] R. C. Batra and G. L. Iaccarino, "Exact solutions for radial deformations of a functionally graded isotropic and incompressible second-order elastic cylinder," International Journal of NonLinear Mechanics, vol. 43, no. 5, pp. 383-398, 2008. 
[20] R. Sburlati and L. Bardella, "Three-dimensional elastic solutions for functionally graded circular plates," European Journal of Mechanics - A/Solids, vol. 30, no. 3, pp. 219-235, 2011.

[21] B. L. Wang and N. Noda, "Thermally induced fracture of a smart functionally graded composite structure," Theoretical and Applied Fracture Mechanics, vol. 35, no. 2, pp. 93-109, 2001.

[22] B. H. Jahromi, H. Nayeb-Hashemi, and A. Vaziri, "Elastoplastic stresses in a functionally graded rotating disk," Journal of Engineering Materials and Technology, vol. 134, no. 2, 2012. 


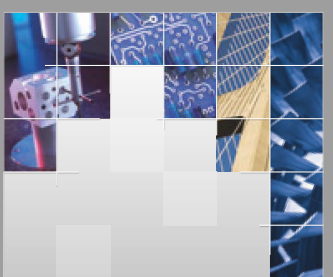

\section{Enfincering}
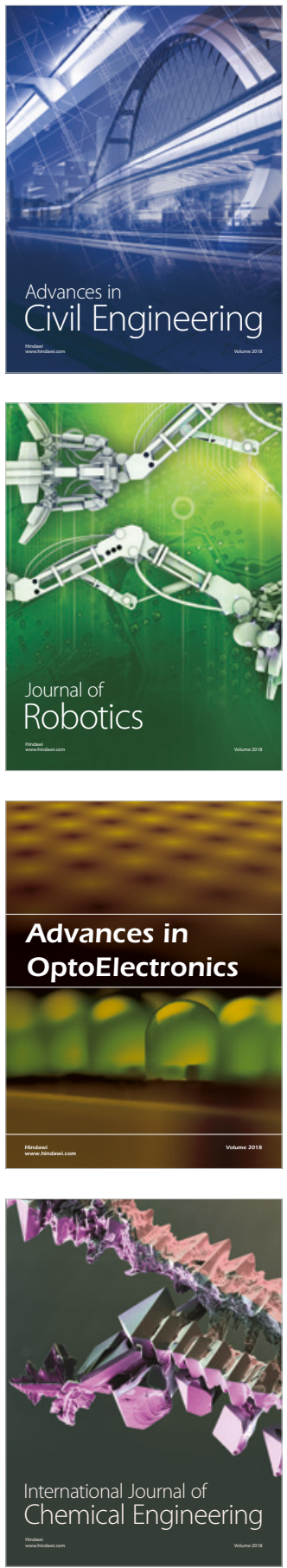

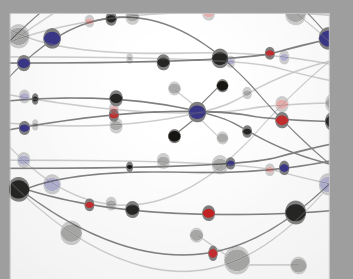

\section{Rotating \\ Machinery}

The Scientific World Journal

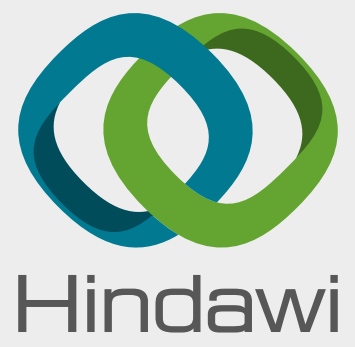

Submit your manuscripts at

www.hindawi.com
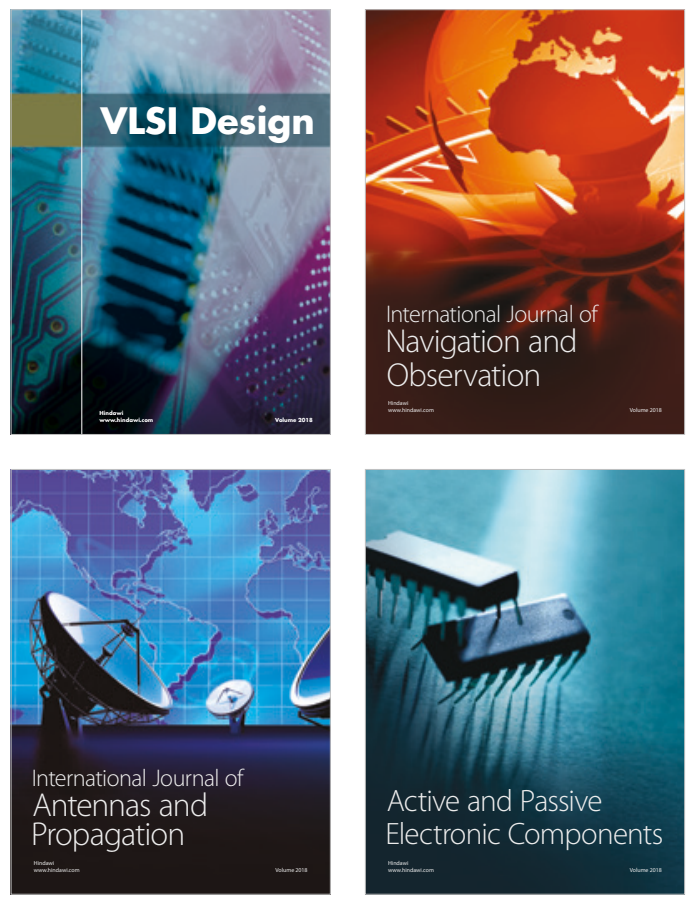
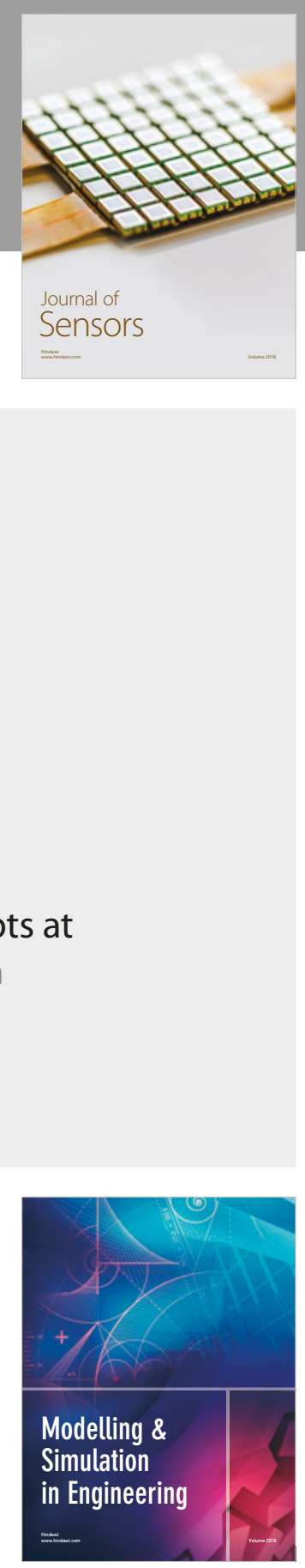

\section{Advances \\ Multimedia}
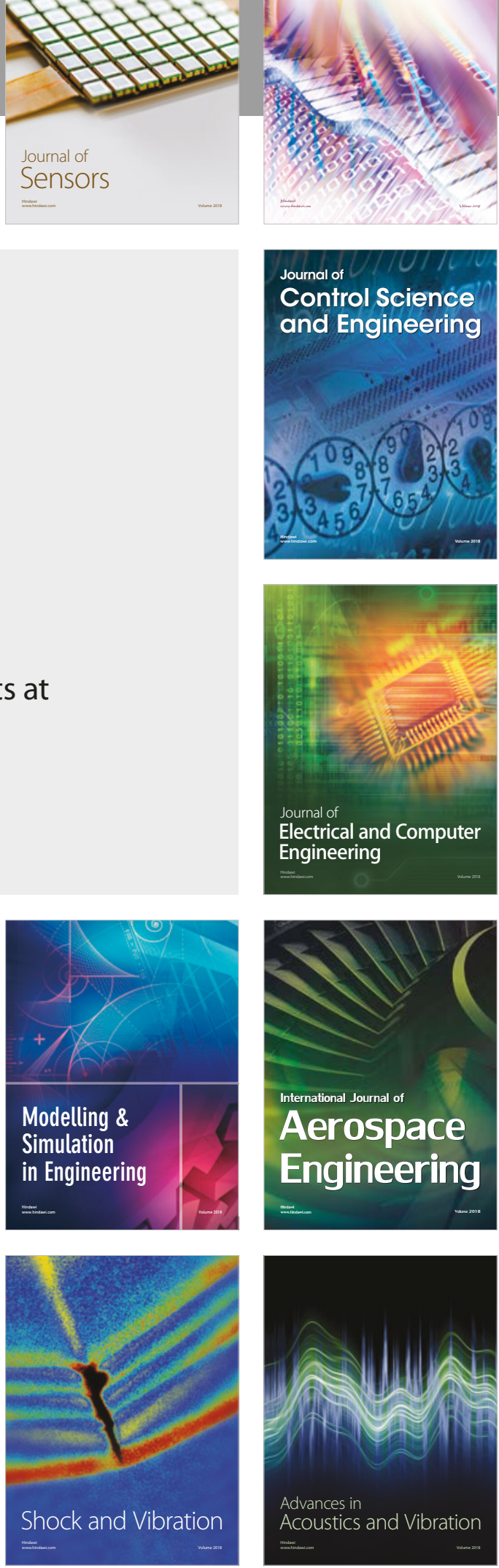